\title{
Palmitoylethanolamide Attenuates Perinatal Asphyxia-induced Damage in the Developing Rat Striatum
}

Lucas D. Udovin ${ }^{1 *} \Delta$, Tamara Kobiec ${ }^{12 \Delta}$, María I. Herrera ${ }^{12}$, Carlos F. Kusnier ${ }^{1}$, Andrea P. Aguilar ${ }^{1}$, Juan P. Luaces ${ }^{1}$, Matilde Otero-Losada ${ }^{1}$ and Francisco Capani ${ }^{13}$

1. Instituto de Investigaciones Cardiológicas (ININCA), UBA-CONICET, CABA, Buenos Aires, Argentina.

2. Centro de Investigaciones en Psicología y Psicopedagogía, Facultad de Psicología, Universidad Católica Argentina, Buenos Aires, Argentina.

3. Departamento de Biología Facultad de Farmacia y Bioquímica, UAJFK.

*. Corresponding author: lucas2304@hotmail.com

${ }^{\Delta}$ These authors contributed equally to this work.

Perinatal asphyxia (PA) is the clinical condition brought by a birth temporary oxygen deprivation associated with long-term damage in the corpus striatum. This is one of the most compromised brain affected areas. Palmitoylethanolamide (PEA) is a neuromodulator well-known for its protective effects in brain injury models including PA, albeit not deeply studied regarding its particular effects in the corpus striatum following PA.

Using the Bjelke et al. (1991) PA model [1], full-term pregnant rats were decapitated, and uterus horns placed in a water bath at $37^{\circ} \mathrm{C}$ for $19 \mathrm{~min}$. One hour later, the pups were injected with PEA $10 \mathrm{mg} / \mathrm{kg}$ s.c. and placed with surrogate mothers. After 30 days, the animals were perfused, and coronal and striatal sections were collected to analyze protein level expression by western blot and the reactive area by immunohistochemistry for neuron markers: Phosphorylated Neurofilament-Heavy/Medium chain (pNFH/M) and Microtubule-Associated Protein-2 (MAP-2) and the astrocyte marker, Glial fibrillary acidic protein (GFAP).

Results indicated that PA produced neuronal damage and morphological changes. Asphyctic rats showed a decrease in pNF-H/M and MAP-2 reactive areas, GFAP+ number cells, and MAP-2 and pNF-H/M protein expression in the striatum. Treatment with PEA partially restored the number of GFAP+ cells, and partially prevented the decrease in $\mathrm{pNF}-\mathrm{H} / \mathrm{M}$ and MAP-2 reactive areas in asphyctic rats. Treatment with PEA also reversed the decrease in MAP-2 protein expression and partially prevented the decrease in pNFH/M protein expression in asphyctic rats. Perinatal asphyxia did not affect GFAP protein level.

Treatment with PEA reduced striatal damage induced by PA, suggesting its therapeutic potential for the prevention of neurodevelopmental disorders.

References:

[1] B Bjelke et al., Brain Res. 543 (1991), p. 1-9. 


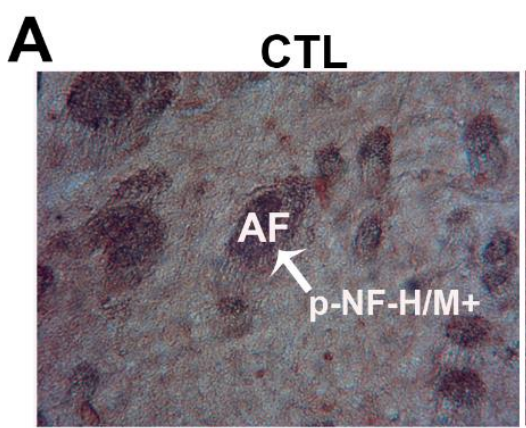

CTL+PEA

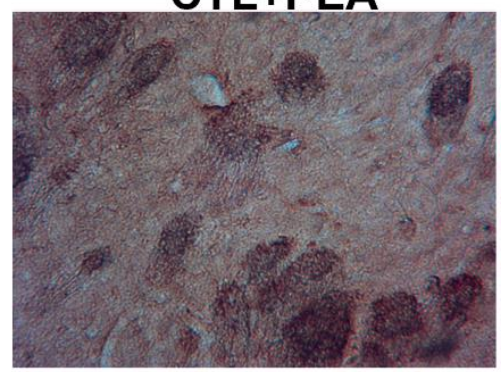

PA

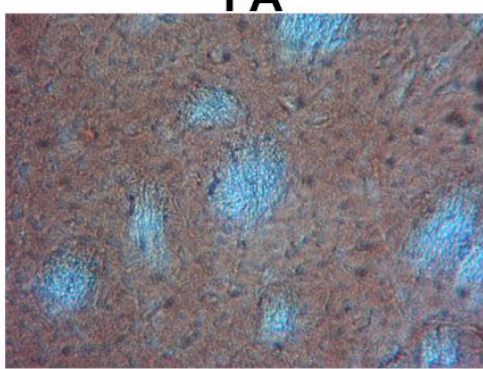

PA+PEA

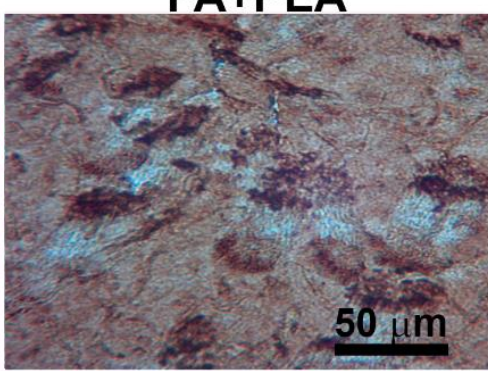

B

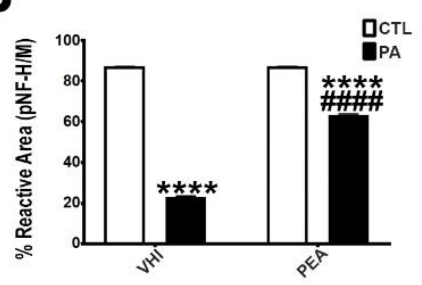

C
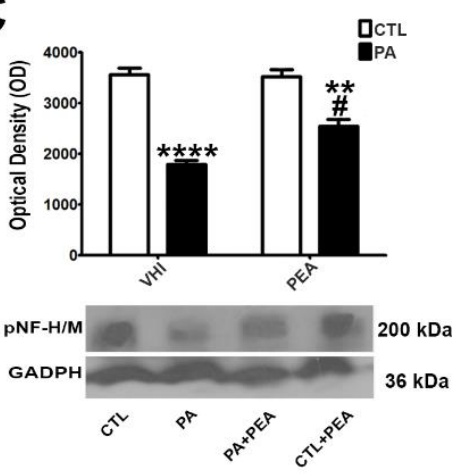

Figure 1. pNF-H/M immunostaining and protein level in the rat striatum. (A) Representative images of the striatum immunostained for $\mathrm{pNF}-\mathrm{H} / \mathrm{M}$ from each experimental group. The white arrow indicates the axonal fascicles (AF). Scale bar: $50 \mu \mathrm{m}$. (B) Percentage of the reactive areas for pNF-H/M $(\mathrm{n}=3),(\mathrm{C})$ Optical density obtained from bands for $\mathrm{pNF}-\mathrm{H} / \mathrm{M}$ protein expression level $(\mathrm{n}=3)$. Bars and error bars represent mean \pm SEM. Two-way ANOVA followed by Tukey post hoc test. **p $<0.01, * * * * p<0.0001$ vs vehicle-treated control group. \#p $<0.05$, \#\#\#\#p < 0.0001 vs PEA-treated PA rats. CTL, Control group; VHI, rats treated with vehicle; PA, rats subjected to PA; PA+VHI, rats subjected to PA and treated with vehicle; PA+PEA, rats subjected to PA and treated with PEA; CTL+PEA, control group treated with PEA. 